Cahiers de recherches médiévales

\title{
Le César de Shakespeare dans son Julius Cassar (1599)
}

Jean-François Chappuit

\section{CpenEdition}

\section{Journals}

Édition électronique

URL : https://journals.openedition.org/crm/2578

DOI : $10.4000 / \mathrm{crm} .2578$

ISSN : 1955-2424

Éditeur

Honoré Champion

Édition imprimée

Date de publication : 30 juin 2007

Pagination : 225-240

ISSN : $1272-9752$

Référence électronique

Jean-François Chappuit, "Le César de Shakespeare dans son Julius Cæsar (1599)», Cahiers de recherches médiévales [En ligne], 14 spécial | 2007, mis en ligne le 30 juin 2010, consulté le 15 décembre 2022. URL : http://journals.openedition.org/crm/2578 ; DOI : https://doi.org/10.4000/crm 2578 


\section{rM}

\section{Le César de Shakespeare dans son Julius Casar (1599).}

La dette de Shakespeare envers Plutarque, dans ses Vies, a été abondamment illustrée ${ }^{1}$. Néanmoins, la critique reste divisée sur le point de savoir si le César dans Julius Caesar est un personnage odieux, thèse surtout défendue par Dover Wilson ${ }^{2}$, ou si, tout en étant ambigu, Shakespeare le montre tout de même sous des aspects favorables, thèse défendue par T.S. Dorsch. Je partirai de l'hypothèse que la dette évidente à Plutarque est l'arbre qui cache la forêt. Shakespeare a suivi la trame narrative des Vies Parallèles ${ }^{3}$, mais il était avant tout l'héritier de l'image traditionnelle de César produite par le Moyen Âge et notamment par Dante. Suétone, dans sa Vie de César (Divus Julius, XXXVII), écrit que, pour son triomphe du Pont, César fit porter devant lui un écriteau avec les trois mots Veni, Vidi, Vici. Je partirai de cette référence célèbre pour mettre en évidence de quelle façon Shakespeare a construit un personnage qui, par ses faits, ses gestes et ses dires, subvertit l'image qu'il a souhaité donner de lui-même et que la postérité a transmise.

\section{Veni, César, homme d'action}

Veni célèbre l'homme d'action ; celui qui traverse le Rubicon et en quelques heures se trouve là où personne ne l'attendait. Les admirateurs de César soulignent à l'envie la force unificatrice qu'il représentait. Shakespeare, en revanche, introduit la notion de rupture dès le premier vers de sa pièce entre les tribuns du peuple et les plébéiens (I, 1, v. 1):

Flavius : Hence! home, you idle creatures, get you home:

Flavius : Hors d'ici, paresseux ! Partez, rentrez chez vous !

\footnotetext{
${ }^{1}$ Voir notamment, T.J.B. Spencer, Shakespeare's Plutarch, Peguin Shakespeare Library, Penguin Books, Harmondsworth, 1964. Voir aussi l'étude de Derek Traversi, Shakespeare: The Roman Plays, Hollis and Carter, Londres, 1963. Pour Derek Traversi il y a une sorte de collaboration entre les deux auteurs : «in the Roman tragedies we are conscious of dealing with what might almost be called a collaboration» (p. 9).

${ }^{2}$ John Dover Wilson (1881-1969) fut responsable de l'édition critique des œuvres complètes de Shakespeare pour le compte des Cambridge University Press. T.S. Dorsch a dirigé l'édition critique de Julius Caesar pour l'édition Arden, Methuen, Londres, 1955. Toutes les citations renverront à cette édition, sauf mention contraire.

${ }^{3}$ L'œuvre de Plutarque Les Vies des hommes illustres ou Vies parallèles, fut traduite du grec en français par Jacques Amyot (1513-1593) et publiée en 1559. Cette traduction française fut ensuite mise en anglais par Thomas North (1535 ?-1601 ?) et publiée en 1579.

${ }^{4}$ Traduction de Louis Lecocq in William Shakespeare, Euvres complètes, édition bilingue, Tragédies I, collection Bouquins, Robert Laffont, Paris, 1995. Toutes les traductions seront de Louis Lecocq sauf mention contraire.
}

Cahiers de Recherches Médiévales, 14spé, 2007 
Chez Plutarque, les citoyens de Rome désapprouvent le triomphe de César; mais Shakespeare modifie sa source. Il met en scène une foule en liesse. Ainsi, l'opposition entre Rome et César, qui se trouve chez Plutarque, est-elle transposée, par Shakespeare, au sein même de Rome. La ligne de fracture est entre la Plèbe et les Tribuns, eux-mêmes plébéiens. Or, ces derniers ont charge de défendre le peuple. Le premier vers de la pièce constitue donc déjà l'image de la nouvelle guerre civile à venir. Les actes de César introduisent le désordre politique et conduisent à la guerre fratricide. Il n'est pas un pacificateur.

Le deuxième aspect mis en avant par Shakespeare est le désordre civil. Les artisans chôment un jour ouvré (I, 1, v. 2). Ils ne portent pas le signe de leur profession. Les lois somptuaires sont bafouées. Les identités ne sont plus reconnaissables. La confusion est à la fois sociale, civile et langagière ; ce qui est mis en lumière par les jeux de mots et les ambiguïtés relationnelles créés autour du mot "cobbler", à la fois « rapetasseur » et «cordonnier». La scène suggère l'affaiblissement de la notion de degré. Cette notion joue encore un rôle important dans la pensée politique élisabéthaine. Elle est aussi liée au principe de plénitude, ce dessein divin selon lequel tous les éléments de la Création sont liés entre eux et cela jusqu'au Créateur; ce qui garantit à l'ensemble son unité et sa stabilité. Par ses actes, César corrompt l'architectonique divine. Il n'est pas bâtisseur mais destructeur. Ce que fit Lucifer au Ciel, César le fait sur Terre.

Cette similitude est corroborée par la notion de béance que Shakespeare introduit vers la fin de la scène. Cette notion est d'abord introduite par celle d'absence ou de vide. Ainsi, Marullus demande (I, 1, v. 32-33) :

\section{Wherefore rejoice? What conquest brings he home? What tributaries follow him to Rome,}

Pourquoi vous réjouir? Quelles sont ses conquêtes? Quels sont les tributaires qui le suivent dans Rome,

La victoire de César n'apporte rien à Rome. La notion d'absence se retrouve un peu plus bas, toujours dans la bouche de Marullus, lorsqu'il évoque avec quelle impatience la Plèbe attendait le passage de Pompée en compagnie de leurs enfants. Ces derniers sont absents pour le triomphe de César. Cette absence est une métonymie de l'avenir de Rome. Elle est reprise dès la scène suivante à propos de la stérilité de Calpurnia, (I, 2). Nous y reviendrons. Le fait que les plébéiens ne soient pas représentés avec leurs enfants nous renseigne aussi sur le but poursuivi par Shakespeare lorsqu'il modifie sa source principale. En effet, Plutarque indique comment les citoyens reprochaient à César d'avoir mis fin à la lignée d'un des plus grands personnages de Rome. Comme pour la notion de division, vue plus haut, Shakespeare reporte donc cette fin de lignée sur le peuple de Rome et, par extension, sur Rome même.

Toujours par le biais de Marullus, Shakespeare représente ensuite la victoire de César comme une chute symbolique. Cette dernière est d'abord illustrée par l'évocation des artisans perchés sur les plus hauts murs, les plus hautes cheminées, au péril de leur vie et de celle de leurs enfants, pour ne voir ne serait-ce que l'ombre de Pompée. Pour le triomphe de César, cependant, la Plèbe est seulement dans la 
rue. Il y a une nette opposition entre les deux représentations. La notion de chute se trouve finalement confirmée par le mot "ingratitude", toujours placé dans la bouche de Marullus (I, 1, v. 53-55):

Run to your houses, fall upon your knees,

Pray to the gods to intermit the plague

That needs must light on this ingratitude.

Rentrez dans vos maisons et tombez à genoux,

Et suppliez les dieux d'arrêter le fléau

Qui va fondre bientôt sur votre ingratitude.

Le mot "ingratitude" est clairement employé dans un contexte religieux. C'est un des mots clefs de la Réforme et chez Calvin cette notion constitue la substance même de la Chute de l'Homme, chute hors de la Grâce de Dieu, dans la fange, la pourriture, le désordre et l'ignorance pour avoir violé l'ordre établi par le Créateur'.

La notion de vacuité apparaît enfin dans la conclusion que Flavius donne à la remontrance de Marullus envers la Plèbe. Pour expurger leur faute, Flavius invite les artisans à se réunir sur les berges du Tibre pour remplir le fleuve de leurs larmes. Le Tibre est ici implicitement vide. Cette image s'oppose à celle de la plénitude du fleuve rempli des cris de joie poussés par la Plèbe à la vue de Pompée (I, 1, 45-47). César est donc associé au vide ; par assimilation, c'est le vide qui s'est ouvert sous les pieds de Lucifer.

Le dernier type de désordre induit par les actes de César concerne la sphère religieuse. Il apparaît à la fin de la première scène de l'acte I. Sur ce point, Shakespeare a également modifié sa source. Il a fait correspondre le triomphe de César, qui eut lieu en octobre 45, et la fête des Lupercales, qui fut fêtée en février 44. Il n'y avait rien de surprenant pour les élisabéthains dans le fait de représenter les sphères politique et religieuse unies l'une à l'autre. Dans l'Angleterre des Tudor, Henry VIII, par l'acte de Suprématie (Act of Supremacy, 1534), était devenu le chef de l'Église Anglicane et fondait en sa personne unique l'État et l'Église de son royaume. Pour ménager les susceptibilités des Catholiques et celles des Puritains, la reine Élisabeth avait changé le mot «chef» par celui de «Governor» dans le compromis des Trente Neuf Articles, compromis adopté en 1563 par le Clergé et en 1571 par le Parlement. Néanmoins, le rétablissement de l'acte de Suprématie signifiait de nouveau que toute atteinte à la majesté de l'une des sphères représentées par le souverain était mécaniquement une atteinte à la seconde. En faisant correspondre

\footnotetext{
${ }^{5}$ Jean Calvin, Institution de la religion chrestienne (édition en français de 1541, traduction par Calvin de l'édition en latin publiée en 1535), texte établi et présenté par Jacques Pannier, deuxième édition Les Belles Lettres, Paris, 1961. Dans l'Epistre au Roy, le mot «ingratitude » se trouve pour la première fois : «En telle manière le Seigneur, quelques eages par cy devant, a puny l'ingratitude des hommes. » (idem, p. 27). Dans le premier chapitre, nous trouvons un des nombreux jugements de Calvin sur l'homme: «Et que feroit l'homme, qui n'est que pourriture et vermine... » (idem, ch. I, «De la Congnoissance de Dieu», p. 42). L'IRC fut traduite en anglais par Thomas Norton et publiée en 1562. La reine Élisabeth a traduit de nombreux passages de l'IRC pour elle-même.
} 
le triomphe de César et la fête des Lupercales, Shakespeare insiste sur un état de chose que tous les Élisabéthains connaissaient et dont ils en comprenaient les conséquences redoutables. Cependant, la création de l'Église Anglicane avait été un acte légal, voté par le Parlement. Cette création constituait donc l'expression de la volonté de tout le peuple anglais. Le souverain représentait l'image de Dieu sur Terre et assurait à son Eglise, sa protection. Avec la modification qu'il a apportée à sa source, Shakespeare suggère plutôt que l'union du politique et du religieux est le fait d'une seule volonté, celle de César. Pour les Élisabéthains, cela faisait de lui un tyran. Ce dernier est toujours coupé du peuple ou de son royaume. Ainsi, César représente-t-il en lui-même la rupture illustrée au tout début de la pièce.

Cette notion, cependant, Shakespeare l'exploite plus avant et de telle façon que les spectateurs en ressentent eux-mêmes l'effet. La conséquence en est l'introduction délibérée d'un aspect de la figure de César qui est dans un rapport anti-thétique à celle qui a été présentée jusqu'alors. Ainsi, tout à la fin de la scène, par le biais de la métaphore du vol égal de tous les oiseaux, Flavius évoque la Rome républicaine, cette Rome de l'égalité des droits et qui serait en danger. Shakespeare passe donc sous silence les ambitions de Pompée et les menaces qu'il faisait peser sur les institutions républicaines de Rome. Plutarque, pourtant, indique clairement que Pompée souhaitait imposer la tyrannie et que, de façon à éviter ce danger, Caton lui-même avait persuadé le Sénat de créer un consulat unique et d'en investir le rival de César. Ainsi, la Rome républicaine évoquée par Flavius n'existe-t-elle plus, en réalité, depuis longtemps.

Par l'évocation du retour à une Rome qui n'existe plus, Shakespeare questionne le concept représenté par le mot «Rome» et, par extension, le concept d'état originaire, ou archaïque au sens propre du terme, et son corollaire, la notion de corps politique. Il questionne le lien que ses contemporains entretiennent avec le passé et la compréhension qu'ils peuvent en avoir. La pièce se caractérise par sa fluidité. Elle s'ouvre sur une scène de passage et se termine de même. C'est le flux inexorable du temps, des époques et des hommes. Shakespeare suggère donc dramatiquement qu'il n'y a pas de restauration possible d'un ordre ancien comme le prétendaient, sur le plan politique, les Tudor, et, sur le plan religieux, les Puritains qui voulaient retrouver l'Église primitive.

D'autre part, le rapport dans lequel il place les deux tribuns par rapport à la Rome qu'ils évoquent reflète le rapport dans lequel il place ses spectateurs par rapport à la Rome qu'il représente. Entre la Rome républicaine et la Rome dominée par César, il y a rupture; et entre l'époque représentée et le monde moderne, il y a une seconde rupture. Deux scènes soulignent cet aspect. La première est celle de l'assassinat, lorsque Shakespeare utilise Cassius pour évoquer les futures représentations de la scène dans les siècles à venir, dans des langues encore inconnues. Cassius s'exprime dans un «temps romain» pour ainsi dire, alors que les spectateurs l'entendent dans un «temps élisabéthains » et, ainsi, ne peuvent pas ne pas être conscients de cette rupture. La seconde est celle de l'éloge funèbre de Brutus prononcé par Marc Antoine. Cet éloge indique la disparition irrévocable d'une époque. L'effet ainsi créé est celui d'une double chute ontologique, l'une après l'autre. En effet, Flavius et Marulus mettent en évidence que la Rome mythique a disparu. C'est la première chute. Cependant, ce monde déchu contient encore César. L'assassinat de ce dernier signale la fin d'un monde qui pouvait produire pareille figure. C'est la 
seconde chute. Le spectateur se trouve donc dans un monde encore appauvri par rapport à la Rome dégradée dont se lamentent les deux tribuns ; ce qui redonne à la figure de César une valeur positive, anti-thétique à celle qui est suggérée tout au long de la pièce et ajoute à la complexité du personnage qui, finalement, n'est pas sans grandeur.

\section{Vidi, César l'observateur, l'auteur des Commentaires}

Le deuxième verbe de la célèbre trilogie nous renvoie à la fois à la perception juste de César et au style précis, net et concis que l'on trouve dans ses écrits et qui fut loué par Cicéron.

Or, la question de voir correctement intervient dès la scène 2, de l'acte I, juste après la référence à la stérilité de Calpurnia. Shakespeare a organisé la scène de façon à ce que l'action progresse du général au particulier. Ainsi, une voix anonyme dans la foule crie le nom de César; puis César demande à cette voix de parler ; ce qu'elle fait: "Beware the Ides of March". César veut alors voir l'homme que Brutus vient de désigner (I, 2, v. 19-21) :

\footnotetext{
Brutus: A soothsayer bids you beware the Ides of March.

Caesar: Set him before me; let me see his face.

Cassius : Fellow, come from the throng; look upon Casar.

Brutus : Un devin qui vous dit: «Crains les ides de mars !»

César: Qu'il vienne devant moi ; je veux voir son visage.

Cassius : Sors de la foule, toi, et regarde César.
}

La mise en garde est énoncée trois fois; d'abord par le Devin, puis par Brutus et, enfin, elle est répétée par le Devin à la demande de César. Ce dernier et le Devin sont alors face à face comme deux miroirs. La démarche d'investigation de César s'apparente à celle de la Scolastique. Elle est circulaire et non progressive. En effet, César demande seulement au Devin de se répéter. César utilise le procédé de l'induction. D'une donnée particulière il tire une vérité générale (I, 2, v. 24) :

Casar : He is a dreamer. Let us leave him. Pass!

César : Cet homme est un rêveur ! Laissons-le. Avancez !

La forte tonalité ironique de ce vers est évidente. Il est entendu que le Devin est aveugle ; mais c'est César qui ne voit pas. D'autre part, nous avons dit que les deux visages sont face à face comme deux miroirs. César occupe donc la place de l'image inversée parce qu'il ne perçoit pas la réalité. Le monde dans lequel il évolue est inversé. Enfin, puisque sa démarche épistémologique est associée à la Scolastique, une école discréditée à l'époque de Shakespeare, César est ainsi associé à un monde dépassé.

Dans la même scène, Shakespeare introduit un autre aspect sensoriel ; celui de la perception auditive. En effet, César dit au vers $17:$ «Speak, Cæsar is turned to hear / Parle ! César s'est tourné pour t'entendre». Cela renvoie à la surdité partielle de César qui est exploitée de façon plus explicite un peu plus bas dans la même 
scène. Nous assistons alors à la sortie du Forum où viennent de se dérouler les jeux. César est soutenu par Marc Antoine. Après lui avoir confié ce qu'il pense de Cassius, il lui demande de venir à sa gauche car son oreille droite est sourde. (I, 2, v. 210-211). Cette surdité ne se trouve pas chez Plutarque. Shakespeare l'a donc délibérément introduite. Elle constitue l'indice non seulement de la partialité politique mais aussi des injures de l'âge et d'une intelligence qui se brouille. Avec cette surdité Shakespeare prolonge le thème de la cécité symbolique de César face au Devin. Une fois encore, César ne peut distinguer ce qui est vrai. Il n'est pas capable de discrétion. Il ne peut distinguer entre les différents éléments qu'il mentionne à propos de Cassius et donne autant d'importance au fait qu'il est maigre et qu'il n'aime pas s'amuser, qu'au fait d'être envieux. Le César de Shakespeare n'est pas l'auteur des Commentaires qui, selon la tradition, relevait avec précision tous les détails de ce qu'il observait.

Shakespeare exploite plus avant l'incapacité de son personnage à distinguer le vrai du faux dans la scène du jardin, chez Brutus (II, 1). Cassius exprime l'inquiétude selon laquelle César est devenu superstitieux et pourrait ne pas se rendre au Sénat (II, 1, v. 193-201). Décius explique alors (II, 1, v. 202-209) comment César croit qu'il est possible de piéger l'unicorne, l'ours, l'éléphant, le lion et l'homme. Pour ce dernier, le piège est la flatterie. Pour convaincre César de se rendre au Capitole, Décius lui rappellera à quel point il déteste les flatteurs, ce qui le flattera beaucoup. Ce n'est pas la première fois que César est associé au monde animal; mais cette association va un peu plus loin ici. Elle fait de lui un être à l'intelligence bornée, incapable de discerner entre le vrai et le faux; donc, comme pour l'animal traqué, incapable de reconnaître les faux branchages qui, à terre, recouvrent le piège et le branchage naturel. Ainsi, César ne distingue pas entre les différents emplois de la flatterie dont Décius fait usage. Cette scène nous renvoie au rapport de vis-à-vis que nous avons relevé plus haut à propos des visages de César et du Devin. Une nouvelle fois Shakespeare représente son personnage comme étant incapable de clairvoyance, un homme qui confond l'image inversée pour celle qui est vraie.

Ce système d'inversion est exploité de façon plus appuyée encore à l'acte I, scène 2 , lorsque Décius entreprend de donner une interprétation favorable au rêve de Calpurnia. Shakespeare met en place un chassé croisé entre le monde du rêve de Calpurnia, monde qui représente la réalité, et le monde de la conscience objective sur lequel s'appuie Décius mais qui est faux. Shakespeare illustre encore ce jeu de miroirs avec l'opposition du verbe "saw" mot au sens concret employé par César pour décrire les sentiments éprouvés par Calpurnia, et le mot «vision» qui, en termes aristotéliciens, représente une substance, et, en tant que tel est un terme qui est sans liaison. Dans les Catégories, Aristote précise que «la substance, au sens le plus fondamental, premier et principal du terme, c'est ce qui n'est ni affirmé d'un sujet, ni dans un sujet $»^{6}$. Lorsque César décrit le rêve de Calpurnia, ce dernier est lié à une réalité politique précise par l'emploi du verbe "saw". Avec le mot «vision» Décius ne relie ce rêve à aucune réalité. Son interprétation représente l'image inversée de celle que César, lui-même, donne au rêve de Calpurnia. C'est cette image inversée

\footnotetext{
${ }^{6}$ Aristote, Catégories 2a, traduction et notes par J. Tricot, Paris, Vrin, 1984, p. 7.
} 
que, une nouvelle fois, César accepte comme l'expression de la vérité. La conclusion de César (II, 2, 91) évoque sa réponse au Devin :

Ceesar: And this way have you well expounded it.

César : Ah ! De cette manière vous l'expliquez fort bien !

Le verbe "to expound" signifie «exposer, expliquer». Il vient du vieux français espondere et du latin exponere : «mettre hors, mettre en vue, étaler, exposer». Le verbe "To expound" connote donc l'unité de l'exposition. Nous retrouvons chez Décius ce que dit Aristote dans De l'Interprétation 16a, à savoir que c'est dans la composition et la division que consiste le vrai et le faux (De l'Interprétation, p ; 78). Une note de Jean Tricot précise : «Dire vrai, c'est dire uni ce qui est uni et séparé ce qui est séparé ; dire faux c'est dire séparé ce qui est uni, et uni ce qui est séparé ${ }^{7}$. L'interprétation de Décius repose sur ce principe aristotélicien et elle est aussi, par extension, scolastique. Revenons à l'exposé que César fait du rêve. Il est implicitement faux, pour ainsi dire, parce qu'il sous-entend que Rome et César ne sont pas unis l'un à l'autre. L'interprétation donnée par Décius, en revanche, est «vraie» pour la raison inverse : elle met en évidence l'union qui existe entre César et Rome. Cette continuité est illustrée, dans le discours de Décius, par l'image du sang de César servant à revivifier Rome.

Les propos de Décius constituent également un discours déclaratif pour la même raison. En effet, sous-jacente à l'interprétation qu'il donne du rêve de Calpurnia se trouve la notion d'unité, notion constitutive du discours déclaratif chez Aristote dans De l'Interprétation 17a : «Est un le discours déclaratif qui exprime soit une chose une, soit une unité résultant de la liaison des parties; par contre, sont composées les propositions qui expriment une multiplicité et non un objet un, ou dont les parties n'ont pas de lien $»^{8}$. Enfin, les propos de Décius se rapportent également à ce que dit Aristote de l'affirmation dans le même traité (17a) : «une affirmation est la déclaration qu'une chose se rapporte à une autre chose ». Ainsi, dans l'interprétation de Décius, César se rapporte naturellement à Rome et inversement. Ce dernier aspect, l'affirmation, se trouve en continuité avec les différents emplois des auxiliaires "shall" et "will" qui sont tous employés par César pour affirmer sa volonté. Shakespeare montre une nouvelle fois que son personnage manque de jugement critique et qu'il a une appréhension globalisante du monde.

César l'observateur, c'est aussi le César rédacteur des Commentaires. Shakespeare y fait brièvement référence avec Cassius dans la scène 2 de l'acte premier (I, 2, v. 124-125):

Ay, and that tongue of his, that bade the Romans

Mark him and write his speeches in their books

Et cette même voix qui disait aux Romains

D'écouter ses discours, d'en prendre note,

${ }^{7}$ Idem, p. 85.

${ }^{8}$ Idem, p. 85. 
Il s'agit ici des faits et gestes de César qu'il dicte aux Romains pour leur servir d'exemple. La netteté et la concision du style des Commentaires sont louées par Cicéron lui-même dans son Brutus (LXXV, 262), et il n'est pas le seul à souligner ces qualités'. César, c'est aussi l'orateur aussi habile que Cicéron, un trait mentionné par Plutarque. Sur ce point encore, Shakespeare prend le contre-pied de la tradition. Le personnage est vantard, et son style est verbeux. Ainsi, lorsque César sort du Forum après les jeux, et qu'il confie à Marc Antoine son opinion sur Cassius, les répétitions sont nombreuses et la pensée suit un chemin circulaire (I, 2, v. 189-211). Le personnage se révèle lui-même conscient du manque de clarté de ses propos. Au début de la scène de l'assassinat, César explicite les paroles qu'il vient d'adresser à Mettelus Cimber à propos de l'exil de son frère (III, 1, v. 39-43). Le personnage utilise des analogies absurdes pour tout lecteur d'Aristote ou de ses commentateurs. Ainsi, à l'acte II, scène 2, César personnifie le danger et prétend être plus dangereux que Danger lui-même. La figure est répétée dans les deux vers qui suivent avec l'image du lion. Le mot «Danger» ici constitue ce qu' Aristote appelle une substance première. À propos de celle-ci Aristote précise dans les Catégories (3b) : «En outre, il semble bien que la substance ne soit pas susceptible de plus et de moins. J'entends par là $[\ldots]$ que toute substance ne peut être dite plus ou moins ce qu'elle est en ellemême; par exemple, cette substance-ci, cet homme-ci, ne sera pas plus ou moins homme que lui-même ou que quelque autre homme ${ }^{10}$. Ainsi, Shakespeare fait dire à son personnage qu'il est plus «danger» que «danger», ou plus «lion» que «lion». Shakespeare fait plus que de mettre en scène sa vantardise, il le ridiculise. La réponse de Calpurnia aux vers de César que nous venons d'examiner met cet aspect en lumière (II, 2, v. 49):

Your wisdom is consum'd in confidence.

Cet excès de confiance éteint votre sagesse.

Le mot "wisdom" suggère la capacité à distinguer entre les différentes catégories du discours. César a oublié ses leçons de rhétorique.

Il en est de même pour la célèbre comparaison avec l'étoile polaire, juste avant l'assassinat (III, 1, v. 60) :

But I am constant as the northern star

Je suis aussi constant que l'étoile polaire

Outre le fait que Shakespeare montre César vacillant sur la question de se rendre au Sénat, dans la scène 2 de l'acte II, cette comparaison est absurde parce qu'il n'existe

\footnotetext{
${ }^{9}$ Voir Bruno Méniel, «César écrivain, d'après les lecteurs de la Renaissance », in La Figure de Jules César au Moyen Âge et à la Renaissance, Cahiers de Recherches Médiévales, ${ }^{\circ} 13$ spécial, année 2006, p. 205.

${ }^{10}$ Aristote, op. cit. p. 16.
} 
pas de relation possible entre un homme et une étoile. N'est comparable que ce qui peut l'être.

À ce point de notre étude, nous voyons que le tableau brossé par Shakespeare est déjà très sombre ; mais ce n'est pas tout.

Vici

Ce verbe connote le conquérant, l'être auquel Dieu donne la victoire, l'homme au-dessus de sa condition. César le conquérant, c'est l'homme qui par la force donne naissance à une nouvelle ère. Ce point est fondamental chez Dante.

Dans la pièce, dès la première apparition de César, à la scène 2 de l'acte II, Shakespeare introduit un élément qui ne se trouve pas chez Plutarque, à savoir la stérilité de Calpurnia. César demande à Marc Antoine de frapper Calpurnia de sa lanière de cuir lors de la course des Lupercales, de façon à chasser la malédiction de la stérilité. La question de cette stérilité renvoie au problème plus général du corps politique et de sa survie. En effet, il y a un parallèle entre l'absence des enfants de la Plèbe dans la première scène de l'acte $\mathrm{I}$, et l'absence de descendance pour César. Ainsi, en raison de l'analogie entre corps politique et corps particulier, la question de la stérilité de Calpurnia renvoie à César lui-même ${ }^{11}$. Si Shakespeare feint d'ignorer la paternité de Brutus, celle de Julia, et finalement celle de Césarion, c'est parce qu'aucun de ces enfants ne lui assure une descendance. La stérilité de Calpurnia symbolise donc l'incapacité de César d'engendrer une dynastie. Shakespeare ne représente donc pas César comme le fondateur d'une ère nouvelle. Cette incapacité à engendrer constitue même l'indice de sa débilité constitutive.

Ce dernier point est développé par Shakespeare dans le deuxième mouvement de cette même scène, acte I, scène 2, mouvement constitué du long dialogue entre Cassius et Brutus, en dehors du Forum où se déroulent les jeux. Cassius raconte d'abord comment César se serait noyé dans le Tibre en crue s'il ne s'était porté à son secours, comme Enée portant son père âgé sur les épaules. Ici encore, Shakespeare modifie sa source principale à propos d'un épisode célèbre et facilement reconnaissable de la guerre de César en Égypte. En effet, Plutarque rapporte comment César, alors tombé à l'eau, réussit à échapper aux forces navales hostiles à Cléopâtre tout en maintenant hors de l'eau quelque livre qu'il avait avec lui. César était donc excellent nageur. Cet épisode se trouve aussi chez Suétone et, plus proche de Shakespeare, chez Sir Thomas Elyot. Le deuxième indice de la faiblesse physique de César est celui du «haut mal» dont il souffrait. Cassius représente, de façon très péjorative, César en proie à une crise d'épilepsie et il le compare à une fillette malade (I, 2, v. 126-130):

Alas it cried, "Give me some drink, Titinius",

A a a sick girl. Ye gods, it doth amaze me

\footnotetext{
${ }^{11}$ Les Élisabéthains étaient très sensibles au problème de la descendance de la reine Élisabeth $1^{\text {ère }}$. Dès son accession au trône, le Parlement lui demanda solennellement de se marier. Elle répondit que son époux était son royaume et ses enfants tous ses sujets. Le parlement ne se contenta pas de cette réponse et renouvela plusieurs fois cette requête; sans succès, comme l'on sait.
} 


\author{
A man of such feeble temper should \\ So get the start of the majestic world, \\ And bear the palm alone. \\ «Hélas ! Je t'en supplie, à boire, Titinius ! » \\ On eût dit qu'une fille souffrait. Je m'étonne, grands dieux, \\ Qu'un homme dont la trempe est si faible l'emporte \\ En majesté sur l'univers, et que lui seul \\ Puisse obtenir la palme !
}

Plutarque, au contraire, mentionne le courage et l'endurance avec lesquels César affrontait ces crises. Le contraste entre la faiblesse de tempérament illustrée par la petite fille malade et l'imperator renvoie à la notion de relation qui est exposée dans les Catégories d'Aristote (6a-6b). Cassius suggère que la corrélation entre les deux éléments ne peut logiquement exister. Une petite fille malade ne peut se trouver au sommet du monde majestueux. Il reste ce que Cassius a objectivement constaté, la faiblesse de tempérament du sujet en question.

La notion de contraste est reprise par Cassius quelques vers plus bas. Cette fois-ci elle s'appuie sur la notion d'opposition entre, d'une part César assimilé au Colosse de Rhodes et, d'autre part, les êtres alentour qui seraient insignifiants, comme Brutus et Cassius (I, 2, v. 133-136). Le contraste est inversé. César est maintenant agrandi et le second terme est diminué ; mais la corrélation n'en est pas moins impossible. César ne peut diminuer par sa stature des hommes tels que Brutus et Cassius parce qu'ils sont tout aussi grands que lui en raison du fait qu'ils sont des «enfants de Rome». Brutus le dit clairement (I, 2, v. 170-173) :

Brutus had rather be a villager

Than to repute himself a son of Rome

Under these hard conditions as this time

Is like to lay upon us.

Brutus aimerait mieux n'être qu'un vil manant

Plutôt que se compter parmi les fils de Rome

Aux dures conditions qu'il semble que ce temps

Veuille nous imposer.

Shakespeare fait ici usage d'une autre catégorie, la qualité. Aristote précise que : «la qualité admet le plus et le moins»(Catégories, 10b). Il ne peut y avoir corrélation entre un César colosse de Rhodes et les autres Romains parce qu'ils sont tous de qualité égale, en tant qu'enfants de Rome. Le premier terme est donc exclu logiquement. L'aspect de la qualité est souligné par Cassius lorsqu'il demande ce qu'il peut y avoir en César lui-même pour faire de lui un être supérieur à Brutus (I, 2, 140):

Brutus and Casar: what should be in that "Casar"?

Brutus et César : qu’y a-t-il donc dans ce «César»? 
Par le biais de Cassius, Shakespeare introduit un autre mode de détermination de la personne, à savoir celui qui repose sur la notion de relation. La personne humaine n'a pas de détermination absolue ; elle n'est définie que par rapport aux éléments qui l'entourent et aussi par rapport à ses actes. Ce procédé de détermination est repris à la scène 3 de l'acte I. Nous y reviendrons. Dans le cas présent, Cassius suggère que la détermination de la personne de César ne résulte pas de la relation entre le Colosse de Rhodes et Brutus, mais entre Brutus et César en tant que fillette malade. Les propos de Cassius, cependant, sont souvent compris comme ceux d'un envieux; ce qui ferait de lui un personnage mesquin. Or, ce n'est pas le cas. Comme presque tous les personnages de la pièce, Shakespeare en a fait un être ambigu, mais sans être méprisable. Shakespeare l'utilise pour diminuer la stature de César et inverser la tradition.

Pour cette dernière, les victoires fulgurantes de César constituent autant de manifestations de la protection divine dont il bénéficiait. Shakespeare sape aussi cette image dès le troisième mouvement de la scène 2 de l'acte $I$, dans le récit que fait Casca de ce qui s'est passé au cours des jeux (I, 2, v. 217-284). Ce récit se situe dans la continuité des propos de Cassius sur la faiblesse physique de César. En effet, pour décrire la crise d'épilepsie de César, Casca utilise le verbe "to swoon", c'est-àdire «perdre connaissance» et «se pâmer». Le verbe s'emploie habituellement pour une femme. Ce pâmoison renvoie donc à l'image de la fillette malade utilisée par Cassius un peu plus haut (I, 2, v. 118-126). Shakespeare va maintenant plus avant. En effet, Casca donne des détails avilissants. César a l'écume à la bouche et il est incapable de parler (I, 2, v. 249-250). Ces détails ne se trouvent pas chez Plutarque. Ici, ils ont pour fonction de ramener César à l'état animal. Shakespeare modifie aussi de cette façon l'interprétation du geste de César après sa crise. En effet, César présente la gorge à tout passant pour qu'elle lui soit tranchée s'il a dit quoi que ce soit d'offensant pour la République. Chez Plutarque, ce geste représente le courage et l'honnêteté de César. Shakespeare en amoindrit l'aspect symbolique pour accentuer la littéralité de la scène. Parce que César a déjà été assimilé à un animal, l'écume à la bouche et incapable de parler, sa gorge tendue fait de lui un bouc émissaire au sens propre du terme. Cette image annonce évidement les débats autour de l'aspect sacrificiel du meurtre de César et de la restauration de la pureté originelle de la Rome républicaine (II, 1, v. 173-180).

Dans la pratique biblique, le bouc émissaire est chargé de tous les péchés et son sacrifice permet de recouvrer la pureté de la Nation et de restaurer l'alliance avec Dieu. Dans le Nouveau Testament, le bouc émissaire a pour image le Christ qui, par son sacrifice, permet l'avènement du Nouvel Adam. Pour la tradition favorable à César, son meurtre représente l'acte sacrificiel qui a précipité l'instauration du nouvel ordre favorable à l'avènement du Christianisme.

Shakespeare, cependant, soulève clairement le problème de la nature du meurtre de César. Au cours de la scène du jardin, après avoir refusé de tuer aussi Marc Antoine, Brutus s'exclame (II, 1, v. 166) :

Let's be sacrificers, but not butchers, Caius.

Soyons, Caius, des sacrificateurs et non pas des bouchers, 
La réponse à cette alternative est implicitement donnée dans les scènes suivantes. Le meurtre de César ne donne pas les résultats escomptés par les conjurés. En conséquence, le terme "sacrificers" se trouve exclu et il ne reste que celui de "butchers". Pris au sens propre, ce terme fait donc, rétroactivement, de César un tyran car ce dernier, en philosophie politique, est toujours assimilé à la bête. Mais Shakespeare n'élimine pas le sens péjoratif du mot "butcher". La question du sacrifice avait une très forte résonance à son époque. En effet, dès le début du $\mathrm{XVI}^{\mathrm{e}}$ siècle, les Protestants soulignent l'aspect païen du sacrifice de l'Eucharistie au cours de la messe catholique. Ils affirment que l'ultime sacrifice est celui du Christ. Avec la référence au bouc émissaire, Shakespeare problématise le modèle biblique de la purification du corps social. Il manifeste, par le jeu dramatique de la pièce, la conscience que les événements ont leur propre dynamique, et que cette dernière est vectorisée sur l'infini. En conséquence, nous retrouvons la notion déjà mise en évidence plus haut et selon laquelle il n'y a pas de restauration possible d'un ordre ancien. Le meurtre de César n'a donc pas de rôle eschatologique ; il est purement politique.

Après la description faite par Casca de la crise d'épilepsie, la question de la détermination de l'identité propre de César est reprise à la scène 3 de l'acte I, dans l'échange entre Cassius et Casca, pendant la tempête. Shakespeare reprend l'association de César au monde animal en rapport avec les idées nouvelles de son époque. Ainsi, par le jeu d'antithèses, il est suggéré que l'identité de César est révélée par ce qui l'entoure. Il peut être loup s'il n'a que des moutons autour de lui, ou lion s'il n'y a que des daims alentour (I, 3, v. 104-106). Ces quelques vers évoquent non seulement la problématique développée par La Boetie dans le De la Servitude volontaire, mais aussi la nouvelle conception de l'identité individuelle et de la vérité qui s'impose graduellement à l'époque. Dans le système ptolémaïque du monde, chaque planète occupe une place déterminée qui est la sienne propre. Il y a correspondance entre l'identité et le lieu occupé. Il en est alors de même dans l'espace social avec la notion de degré. À la Renaissance, ce système est remis en cause tant par les découvertes géographiques que par les progrès technologiques, scientifiques et culturels. De même que les astronomes découvrent que l'espace est infini et que la position d'un corps céleste ne peut se définir de façon absolue mais seulement par rapport à d'autres points de références, eux-mêmes en mouvement, de même dans l'espace social, vérité et identité ne peuvent être déterminées que de façon relative et non pas absolument. Ces quelques vers prononcés par Cassius constituent donc un modèle de détermination des personnages. Cela explique l'interjection "Poor man!" que Shakespeare lui fait prononcer. César n'est rien de façon absolue, rien de ce qu'il croit être par lui-même. Ce qu'il est dépend de ce qui l'entoure. Shakespeare problématise la notion d'individu ; c'est-à-dire ce qui fait qu'un individu est ce qu'il est. Un individu se détermine par le jeu de relations entre la nature qu'il a reçue du fait de sa naissance, du rapport qu'il entretient avec cette nature, et du rapport que cet ensemble entretient avec le monde.

Enfin, César fut divinisé en 26 avant Jésus-Christ. César est immortel. Dans la Divine Comédie, Dante exploite cette notion, mais à propos de l'esprit de César, esprit qui est celui du gouvernement unique, celui de la monarchie. Dans le chant VI 
du Paradis (v. 55-85), le récit passe de Jules César à César Auguste sans transition ${ }^{12}$. La monarchie est immortelle en esprit. Shakespeare fait allusion à ce point lorsque Brutus s'exclame (II, 1, v. 166-170) :

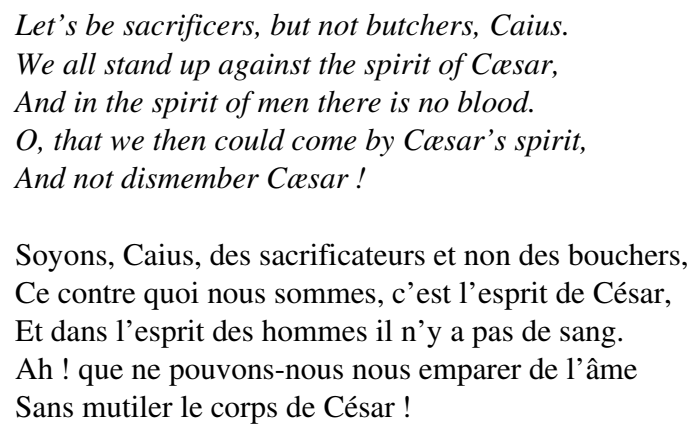

Soyons, Caius, des sacrificateurs et non des bouchers, Ce contre quoi nous sommes, c'est l'esprit de César, Et dans l'esprit des hommes il n'y a pas de sang. Ah ! que ne pouvons-nous nous emparer de l'âme Sans mutiler le corps de César !

Shakespeare utilise la notion de double personne du roi, à la fois mortelle corporellement, et immortelle dans le principe politique qu'elle incarne ${ }^{13}$. Shakespeare n'a donc pas omis d'inclure dans son personnage l'aspect divin que la tradition lui reconnaît.

Cet aspect est traité à l'aide de l'association des mots "cause", "will" (la volonté), et de l'adjectif "constant". Les deux premiers termes apparaissent ensemble au cours de la scène 2 de l'acte II, chez César. À Décius qui lui demande la cause de son refus de se rendre au Sénat, César répond (II, 2, v. 74) :

The cause is in my will : I will not come.

La cause est dans ma volonté ; je ne veux pas.

Shakespeare replace ensuite le mot «cause » dans la bouche de César, au Sénat, alors que Metellus Cimber lui demande de rappeler son frère de l'exil (III, 1, v. 47-48) :

Know, Casar doth not wrong, nor without cause

Will he be satisfied.

César ne fait du tort qu'avec juste raison,

Et sans bonne raison on ne le convainc pas.

Ces vers furent moqués par Ben Jonson et ils ont été l'objet de nombreux commentaires. À mon sens, Shakespeare utilise la définition de Dieu qui se trouve chez Aris-

\footnotetext{
${ }^{12}$ Dante, Divine Comédie, Paradis, (chant VI, 55-85), in CEuvres complètes, traduction et commentaires par André Pézard, Bibliothèque de la Pléiade, Paris, Gallimard, 1965, p. 14051407.

13 Voir Ernst H. Kantorowicz, The King's Two Bodies, A Study in Medieval Political Theology, Princeton, New Jersey, Princeton University Press, 1957.
} 
tote déjà et qui sera reprise par de nombreux auteurs chrétiens dont saint Thomas d'Aquin. Premièrement, Dieu est bon. Ainsi, César, assimilé à Dieu, ne peut faire le mal ou ce qui est mauvais, c'est-à-dire, dans les vers cités plus haut, "wrong". D'autre part, Dieu est cause efficiente. Il est, selon les termes d'Aristote, le Premier Moteur, et il induit le désir de ce qui est bon en tous les étants. César dit donc qu'il ne voit pas de cause induite chez le banni, c'est-à-dire de mouvement du banni vers lui. En conséquence il n'y a pas de cause de le rappeler. C'est le banni qui doit être mû. Le personnage affirme donc son essence divine. Cela est encore évident lorsqu'il se compare à l'étoile polaire (III, 1, v. 60). À propos du temps, dans les Catégories 5a), Aristote écrit: «comment ce qui n'est pas permanent pourrait-il avoir une position? » La similitude avec l'étoile polaire indique donc deux autres caractéristiques divines du personnage : il occupe une seule position et il est hors du temps.

Le personnage ne fait donc pas de distinction entre les notions de cause et d'effet parce qu'il s'affirme cause efficiente de tout; ce qui est la caractéristique de l'essence divine ${ }^{14}$. Cependant, au cours de la scène 2 de l'acte II, scène qui précède de peu celle de l'assassinat, Shakespeare multiple les indices qui révèlent l'inconstance du personnage et questionne ainsi tout aspect divin de sa personne. Dès le début de la scène (II, 2), il donne à son personnage la pleine conscience du danger qu'il court puisqu'il lui fait répéter les mots prononcés par Calpurnia dans son sommeil :

\section{Thrice hath Calpurnia in her sleep cried out \\ "Help, ho! They murther Casar!" \\ Par trois fois en dormant Calpurnia \\ A crié « À l'aide ! Ha ! On assassine César ! »}

Calpurnia fait ensuite son entrée et interdit à César, de façon catégorique, de sortir, (II, 2, v. 8-9). Cette interdiction se fait par le jeu du modal "shall" qui, en anglais, exprime une contrainte sur le sujet. "Shall" s'oppose à l'auxiliaire "will" qui, lui, exprime la volonté propre du sujet. Dans toute la scène, Shakespeare exploite ces deux nuances. Ainsi, le "You shall not stir" (II, 2, 9) trouve son symétrique dans le vers prononcé par César vers la fin de la scène : "Give me my robe, for I will go" (II, 2, v. 107). Cet emploi des modaux illustre la prétention du personnage à échapper à la vicissitude. Donc, à l'interdiction de Calpurnia, la réponse de César est d'affirmer par trois fois, et sous la même forme, qu'il sortira : "Cæsar shall forth" (v. 10), "Yet Cæsar shall forth" (v. 28), et finalement, au vers 48: "And Cæsar shall go forth". Cependant, le personnage finit par accéder à ce qu'il appelle le «caprice» en anglais "humour" de Calpurnia,: "And for thy humour I will stay at home" (II, 2, v. 56). "I will stay" signifie : «je décide, moi, de rester ».

Shakespeare met donc en scène l'absence de constance chez César sur deux plans. Le premier plan est celui de la simple contradiction de lui-même. Le second

\footnotetext{
${ }^{14}$ À l'époque de Shakespeare, cette notion est également au cœur de la doctrine de la prédestination chez Calvin et de la controverse à propos du libre arbitre. Mais cette remarque concerne plutôt Brutus et sort du cadre du sujet.
} 
plan est celui des causes qui motivent les décisions de César. Il refuse de reculer face aux complots (v. 10 à 12), face à la destinée (v. 26 à 29), face à la mort (v. 32 à 37), face aux dieux et aux dangers du monde (v. 41 à 48). Tout cela est ensuite balayé par ce que César qualifie de «caprice». Ce déséquilibre illustre la démesure du personnage au sens aristotélicien du terme. Il y a d'une part excès puis insuffisance dans les raisons qui motivent ses décisions. Le déséquilibre est aussi présent structurellement par la juxtaposition du ressort comique des refus successifs et le tragique de la situation ; tragique qui est réaffirmé au début de la scène et qui est présent dans l'esprit de tous les spectateurs. Shakespeare exploite plus avant cet élément de comédie tragique avec l'entrée de Décius. Il y a un renversement de situation. Cette fois, César refuse par trois fois de se rendre au Sénat: "And tell them I will not come today" (II, 2, v. 62), puis, au vers 68 : "Decius, go tell them I will not come"; enfin, au vers 71 : "I will not come". Cependant, après l'interprétation que Décius fait du rêve de Calpurnia, (II, 2, v. 83-90), César change brusquement d'avis : "I will go" (II, 2, v. 107). Ce qui a été dit plus haut de la démesure du personnage, de son hybris, est donc répété ici ; c'est le signe de l'importance que Shakespeare donne à cet aspect de son personnage. La personne de César n'est pas divine.

Shakespeare révèle aussi la nature instable de son personnage avec le traitement du prétexte qui doit justifier le refus de se rendre au Sénat. Son évolution graduelle épouse savamment le déroulement de l'action. Ainsi, lorsque César cède aux injonctions de Calpurnia, il reprend à son compte le prétexte qu'elle a suggéré, à savoir envoyer Marc Antoine dire au Sénat que César est malade. César reprend à son compte cette suggestion avec le modal "shall", ce qui indique qu'il endosse entièrement la portée de ce mensonge (II, 2, v. 55). Décius entre sur scène, et César ment par omission. Puis, lorsque Calpurnia demande à Décius de dire au Sénat que César est malade, ce qui est consistent avec ce qui a précédé entre elle et César, ce dernier s'indigne (II, 2, v. 65-67):

\author{
Shall Casar send a lie? \\ Have I in conquest strech'd mine arm so far, \\ To be afeard to tell greybeards the truth? \\ César leur mentirait? \\ Ai-je de mon seul bras conquis tant de contrées \\ Pour n'oser parler franc devant des barbes grises?
}

En l'espace de dix vers, le personnage inverse sa position. Cette image de renversement est reprise immédiatement après dans les vers 71 à 74 . En effet, César se contredit. Il affirme d'abord à Décius que la cause de son refus est issu de sa volonté propre (II, 2, v. 71), puis il lui explique que la raison est Calpurnia elle-même. À la fin de la scène, il change d'avis pour la troisième fois quant à la question de se rendre au Sénat. Nous trouvons ainsi le chiffre neuf.

Pour le dernier point de mon exposé, revenons aux vers 65-67, II, 2 cités plus haut: "Shall Cæsar send a lie ?". L'aspect le plus intéressant de ces trois vers réside dans l'opposition entre les mots "lie" (mensonge) et "truth" (vérité). Dans le Livre de la Genèse, Dieu sépare la Lumière des Ténèbres, le Ciel de la Terre. La Création se caractérise par la fin du Chaos, l'avènement d'un espace ordonné. Ce thème est 
repris lorsque Dieu conduit les animaux auprès d'Adam pour que ce dernier les nomme. Adam, par la parole, ordonne aussi. Dans la scène que nous étudions, nous voyons que Shakespeare fait se confondre mensonge et vérité chez son personnage. Cela fait de ce dernier un être amoral ; aspect qui se trouve chez Plutarque, notamment à propos de la saisie du Trésor Public. Shakespeare associe alors César au Chaos ; et non au Créateur qui ordonne le monde pour le faire accéder à l'existence.

Nous venons donc de voir comment Shakespeare subvertit délibérément la figure traditionnelle de César héritée principalement du Moyen Âge. Mais cela nous amène à une autre constatation. Si Shakespeare a bien suivi Plutarque à la lettre, pour ainsi dire, il en a aussi suivi l'esprit. En effet, dans sa brève introduction à la Vie d'Alexandre le Grand, Plutarque écrit : «je n'ai pas appris à écrire des histoires mais des vies seulement; et les plus hauts et les plus glorieux exploits ne sont pas toujours ceux qui montrent mieux le vice ou la vertu de l'homme; mais bien souvent une légère chose, une parole ou un jeu, mettent plus clairement en évidence le naturel de personnes, que ne font pas des défaites où il sera demeuré dix mille hommes morts, ni les grosses batailles, ni les prises de villes par siège ni par assaut ». C'est ce que Shakespeare a fait ; il a créé un personnage qui se révèle lui-même par nombres d'indices qui sont sans apparente grande importance. Cependant, cette représentation est aussi en rapport avec le contexte socio-politique de l'époque. César, c'est aussi la puissance de l'État, à la fois indispensable et redoutable. Mais le sens véritable du personnage se déduit de la fonction que Shakespeare lui fait jouer vis-à-vis de l'autre grande figure de la pièce, c'est-à-dire Brutus. Ce dernier est tout aussi complexe et paradoxal, et les ambiguïtés de l'un se reflètent chez l'autre jusqu'à la fin de l'œuvre lorsque Brutus dépasse cette opposition et que César devient, paradoxalement, la pierre de touche de ce dépassement. Mais cela est un autre sujet.

Jean-François Chappuit MCF Versailles Saint Quentin en Yvelines 